\title{
Derivation and Application of an Algorithm for the Numerical Calculation of the Local Orientation of Nematic Liquid Crystals
}

\author{
A. Kilian and S. Hess \\ Institut für Theoretische Physik, Technische Universität Berlin, Berlin
}

Z. Naturforsch. 44a, 693-703 (1989); received June 16, 1989

\begin{abstract}
Starting from a relaxation equation for the alignment tensor, an algorithm is derived which allows the numerical calculation of the dynamic and static behavior of the director field $\mathbf{n}$ with the correct nematic symmetry property, where $\mathbf{n}$ and $\mathbf{- n}$ are equivalent. As a first application, a two-dimensional problem is treated where the typical nematic defects with half-integer winding numbers only occur when the algorithm with the correct nematic symmetry property is used. Furthermore, the method is applied to the static and dynamic behavior of a Frederiks cell with strong and weak anchoring.
\end{abstract}

\section{Introduction}

Simple differential equations can posses rather complicated solutions. This also applies to the equations of nemato-dynamics [1-3] governing the director $\mathbf{n}$ which describes the local orientation of nematic liquid crystals. Analytic solutions can often just be obtained for rather special cases. Thus it is desirable to use numerical solution procedures for the application of the fundamental equations to display devices [4, 5]. Numerical methods are also of interest from a mathematical point of view [6] and for the understanding of the rich physical phenomena occurring when the molecular orientation is subjected to the competing influences of external fields, of walls and of flow processes [7].

It is the purpose of this article to present a numerical method for the analysis of the static and dynamical behavior of the director field $\mathbf{n}$ of a nematic liquid crystal, where the physical equivalence of $\mathbf{n}$ and $-\mathbf{n}$ is taken into account. This point is of little concern for analytic calculations where the solutions with the correct symmetry can be selected by inspection or by making an appropriate ansatz. A numerical method designed to be applicable to one-, two-, and three-dimensional problems, however, has to take the correct symmetry of "order parameter space" into account since the topological properties of defects are affected by the symmetry [8]. In particular, defects with half-in-

Reprint requests to Prof. Dr. S. Hess, Technische Universität Berlin, Institut für Theoretische Physik, Sekr. PN 7-1, Hardenbergstr. 36, D-1000 Berlin 12, West-Germany. teger winding numbers to which the nematic threads are attached [1, 2] occur only when $\mathbf{n}$ and $-\mathbf{n}$ are equivalent.

For simplicity and in order to focus on the essential points, the coupling between orientation and flow is disregarded and the elasticity of nematics is treated in the one-coefficient approximation. Two specific applications of the numerical method are discussed. The first one is a two-dimensional boundary value problem which shows the expected differences between results obtained with the new "tensorial" algorithm and those found with the standard "vectorial" method. The second example, viz. the determination of the Frederiks transition for a one-dimensional problem, where a (partial) comparison with the analytic results is possible, is meant to demonstrate the reliability of the numerical procedure.

This article proceeds as follows. The derivation of the algorithm is outlined in section 2. Point of departure is a relaxation equation for the (second rank) alignment tensor $[9,10]$ which allows a unified description of nonequilibrium alignment phenomena both in the isotropic and the nematic phases of a liquid crystal. The standard equations of elasto-nemato-dynamics are recovered if one assumes the alignment tensor to be uniaxial and to posses a constant magnitude which is essentially the Maier-Saupe order parameter $S$. Furthermore, the tensorial equation is reduced to the usual vectorial equation of the director field $\mathbf{n}$ by a projection. It is demonstrated that the discretization of the spatial derivatives needed for the numerical computations must be made prior to this projection if one wants to preserve the nematic

0932-0784/89/0800-0693 \$ 01.30/0. - Please order a reprint rather than making your own copy. 
symmetry inherent in the tensorial description, i.e. the equivalence of $\mathbf{n}$ and $-\mathbf{n}$.

The stationary solutions of the relaxation equation correspond to minima of a free energy functional. The discretization of this quantity is also discussed.

Section 3 is devoted to the above mentioned applications.

\section{Derivation of the Algorithm}

\subsection{Relaxation Equation for the Alignment Tensor}

The orientation of (effectively) uniaxial molecules with their figure axis parallel to the unit vector $\mathbf{u}$ is characterized by the (second rank) alignment tensor

$$
a_{\mu v}=\zeta\left\langle\overleftrightarrow{u_{\mu} u_{v}}\right\rangle
$$

Cartesian components are denoted by Greek subscripts, the summation convention is used for them. The bracket $\langle\ldots\rangle$ indicates an average (to be evaluated with the one-particle distribution function). The symbol $\leftrightarrow$ refers to the symmetric-traceless (irreducible) part of a tensor t, e.g.

$$
\overleftrightarrow{t_{\mu v}}=\frac{1}{2}\left(t_{\mu v}+t_{v \mu}\right)-\frac{1}{3} t_{\lambda \lambda} \delta_{\mu v},
$$

where $\delta_{\mu v}$ is the unit tensor. The factor $\zeta$ occurring in (1) can be chosen conveniently, its specification is not needed here. Of course, measurable properties are independent of $\zeta$. In an uniaxial state the alignment tensor reduces to

$$
a_{\mu v}=\sqrt{\frac{3}{2}} a \overleftrightarrow{n_{\mu}} n_{v}
$$

where the unit vector $\mathbf{n}$ is the director. The "magnitude" (modulus) of the alignment tensor is

$$
a=\sqrt{\frac{2}{3}} \zeta S,
$$

where $S=\left\langle P_{2}(\mathbf{n} \cdot \mathbf{u})\right\rangle$ is the Maier-Saupe order parameter. The factor $\sqrt{\frac{3}{2}}$ has been inserted in (3) such that $a_{\mu \nu} a_{\mu v}=a^{2}$.

Coupled nonlinear relaxation equations for the alignment tensor have been derived within the framework of irreversible thermodynamics [9-11] and by starting from a generalized Fokker-Planck equation [12]. These equations allow a unified treatment of nonequilibrium phenomena in the isotropic and the nematic phases; the de Gennes theory for the pretransitional behavior [1,2] and the Ericksen-Leslie theory for nematics are special cases of the unified theory. If the coupling with the flow field is disregarded for sim- plicity, the relaxation equation for $a_{\mu v}$ reads

$$
\tau_{\mathrm{a}} \frac{\partial a_{\mu v}}{\partial t}-\xi_{0}^{2} \Delta a_{\mu v}+\Phi_{\mu v}-F_{\mu v}=0 .
$$

Here $\tau_{\mathrm{a}}$ is a (phenomenological) relaxation time coefficient; $\xi_{0}$ is a bare correlation length (typically of the order of the length of a molecule), and $\Delta=\nabla_{\lambda} \nabla_{\lambda}$ is the Laplacian. In general, additional terms [13] involving spatial derivatives $\nabla$ of the alignment tensor have to be included in (5) in order to recover the full anisotropy of the Frank elasticity involving three coefficients $K_{i}$. For simplicity, the present analysis is restricted to the one-coefficient approximation with $K_{1}=K_{2}=K_{3}$. The quantity

$$
\Phi_{\mu v}=\frac{\partial \phi}{\partial a_{\mu v}}
$$

is the derivative of a (dimensionless) Landau-de Gennes potential $\phi$ depending on the scalar invariants of $a_{\mu v}$. A specific expression for $\phi$ is not needed here. The free energy density associated with the alignment is

$$
f^{\mathrm{LG}}=\frac{\varrho}{m} k_{\mathrm{B}} T \phi,
$$

where $Q$ is the mass density of the fluid, $m$ the mass of a molecule, $k_{\mathrm{B}}$ the Boltzmann-constant, and $T$ the temperature. The tensor $F_{\mu v}$ is describing the influence of an orienting external field; e.g. for molecules with an anisotropic magnetic susceptibility in the presence of a magnetic field $\mathbf{B}$, one has

$$
F_{\mu v}=\frac{1}{2}\left(\frac{\varrho}{m} k_{\mathrm{B}} T\right)^{-1} \xi^{-1}\left(\chi_{\mathrm{par}}-\chi_{\mathrm{per}}\right) \mu_{0}^{-1} \overleftrightarrow{B_{\mu} B_{v}}
$$

Here, $\chi_{\text {par }}$ and $\chi_{\text {per }}$ are the susceptibilities parallel and perpendicular to the molecular symmetry axis for a perfectly oriented sample $(S=1) ; \mu_{0}$ is the magnetic permeability coefficient of the vacuum. Note that $\chi$ here is defined by $M_{\mu}=\mu_{0}^{-1} \chi_{\mu \nu} B_{v}$ rather than by $M_{\mu}=\chi_{\mu v} H_{v}$ [3]. For an electric field $\mathbf{E}$ instead of a magnetic field $\mathbf{B}, \mu_{0}^{-1}\left(\chi_{\text {par }}-\chi_{\text {per }}\right)$ is replaced by $\varepsilon_{0}\left(\varepsilon_{\mathrm{par}}-\varepsilon_{\mathrm{per}}\right)$ where $\varepsilon_{0}$ is the dielectric permeability of the vacuum, and $\varepsilon_{\text {par }}, \varepsilon_{\text {per }}$ are relative dielectric coefficients. The change of $a^{2} \equiv a_{\mu v} a_{\mu v}$ with time can be inferred from (5) after multiplication by $a_{\mu v}$. In a stationary and spatially homogeneous state and in $a b-$ sence of external fields this equation reduces to

$$
\frac{1}{2} \frac{\mathrm{d}}{\mathrm{d} t} a^{2} \equiv a_{\mu v} \frac{\partial a_{\mu v}}{\partial t}=a_{\mu v} \Phi_{\mu v}=0 .
$$


Since $\phi$ is a nonlinear function of the alignment tensor (1), (2), this equation yields the equilibrium value $a_{\mathrm{eq}}$ for the order parameter $a$, cf. (3).

In the nematic phase of thermotropic liquid crystals, the magnitude $a_{\text {eq }}$ is practically not affected by applied fields [14]. External fields, however, exert torques which are discussed next in connection with the local orientation.

\subsection{Equation of Change for the Director}

Directional changes can be inferred from (5) after multiplication by $C \cdot \varepsilon_{\lambda \varkappa \mu} a_{\varkappa v}$, where $\varepsilon_{\lambda \varkappa \mu}$ is the totally antisymmetric isotropic tensor of rank three (with it, the cross product of two vectors $\boldsymbol{b}$ and $\boldsymbol{c}$ reads $\left.(\boldsymbol{b} \times \boldsymbol{c})_{\lambda}=\varepsilon_{i \times \mu} b_{\varkappa} c_{\mu}\right)$. The factor $C$ can be chosen conveniently. The resulting equation is

$$
C \cdot \varepsilon_{\lambda \times \mu} a_{\varkappa v}\left(\tau_{\mathrm{a}} \frac{\partial a_{\mu v}}{\partial t}-\xi_{0}^{2} \Delta a_{\mu v}-F_{\mu v}\right)=0
$$

since $\varepsilon_{i \varkappa \mu} a_{\varkappa v} \Phi_{\mu v}=0$. If one now assumes that the tensor $a_{\mu v}$ is of the uniaxial form (3) and that the magnitude of $a$ is given by its constant equilibrium value $a_{\mathrm{eq}}$, (10) reduces to

$$
\varepsilon_{\lambda \varkappa \mu} n_{\varkappa} W_{\mu}=0
$$

with

$W_{\mu}=n_{v}\left[\gamma_{1} \frac{\partial}{\partial t}\left(n_{v} n_{\mu}\right)-K \Delta\left(n_{v} n_{\mu}\right)-\chi_{\mathrm{a}} \mu_{0}^{-1} \overleftrightarrow{B}_{\mu} B_{v}\right]$

Due to the choice of $C=2 \frac{\varrho}{m} k_{\mathrm{B}} T$, which is determined by an analysis of the free energy (Sect. 2.4), the Leslie coefficient $\gamma_{1}$, the Frank elasticity coefficient $K$, and the susceptibility coefficient $\chi_{\mathrm{a}}$ associated with the molecular anisotropy are related to the coefficients $\tau_{\mathrm{a}}$, $\xi_{0}$, and $\chi_{\text {par }}-\chi_{\text {per }}$ by

$$
\begin{aligned}
& \gamma_{1}=3 a_{\mathrm{eq}}^{2} \tau_{\mathrm{a}} \frac{\varrho}{m} k_{\mathrm{B}} T, \\
& K=3 a_{\mathrm{eq}}^{2} \xi_{0}^{2} \frac{\varrho}{m} k_{\mathrm{B}} T, \\
& \chi_{\mathrm{a}}=\sqrt{\frac{3}{2}} a_{\mathrm{eq}} \zeta^{-1}\left(\chi_{\mathrm{par}}-\chi_{\mathrm{per}}\right)=S\left(\chi_{\mathrm{par}}-\chi_{\mathrm{per}}\right) .
\end{aligned}
$$

The special choice of $\zeta=\sqrt{\frac{15}{2}}$ used in (9)-(13) leads to $a_{\mathrm{eq}}^{2}=5 S^{2}$.

Use of $n_{v} \frac{\partial}{\partial t} n_{v}=0$ and

$$
\varepsilon_{i \times \mu} n_{\varkappa}\left(n_{v} \Delta\left(n_{v} n_{\mu}\right)-\Delta n_{\mu}\right)=0
$$

due to $n_{v} n_{v}=1$ leads to the standard "vectorial" expression

$W_{\mu}^{\mathrm{V}}=\gamma_{1} \frac{\partial n_{\mu}}{\partial t}-K \Delta n_{\mu}-\mu_{0}^{-1} \chi_{\mathrm{a}} n_{v} B_{v} B_{\mu}$,

which is linear in $\mathbf{n}$. Solution of the equation $W_{\mu}^{\mathbf{V}}=0$ subject to the constraint $n_{\mu} n_{\mu}=1$ is the usual way to treat nemato-dynamics (in the one-elasticity coefficient approximation).

In the mathematical literature $[6,15]$, the study of the equation $\Delta \mathbf{n}=0$ with the constraint $n_{\mu} n_{\mu}=1$ is often called the "liquid crystal problem". As already mentioned in the introduction, the physical equivalence of $\mathbf{n}$ and $-\mathbf{n}$ is not apparent in the standard approach. In the vectorial description, which is more appropriate for a ferro-magnetic system, $\mathbf{n}$ can be represented by a point on the two-sphere $S^{2}$. For the true nematic symmetry it is to be represented by a point on the projective two-plane $P^{2}$. Moreover, it was recently shown that there are conceptional problems with the derivation of a macroscopic director from an ensemble of molecular directors within the framework of statistical physics [16].

Next, it is demonstrated that a spatial discretization needed for a numerical method which starts from (12) rather than from (15) maintains the nematic symmetry.

\subsection{Spatial Discretization}

For the numerics, the rectangular coordinates $r_{\mu}$, $\mu=1,2,3$ are discretized according to $r_{\mu}=i_{\mu} \delta l$ with integers $i_{\mu}$. The length $\delta l$ is the distance between the lattice points. The second spatial derivative $\frac{\partial^{2}}{\partial r_{1}^{2}}$ of a function $G$ at the point $i$ is approximated by

$$
\begin{aligned}
\frac{\partial^{2}}{\partial r_{1}^{2}} G \approx & \delta l^{-2}\left[G\left(i_{1}+1, i_{2}, i_{3}\right)+G\left(i_{1}-1, i_{2}, i_{3}\right)\right. \\
& \left.-2 G\left(i_{1}, i_{2}, i_{3}\right)\right]
\end{aligned}
$$

This expression follows from an expansion of the function $G$ at the lattice positions with $i_{1} \pm 1, i_{2}, i_{3}$ around $i_{1}, i_{2}, i_{3}$, where terms of the order $\delta l^{4}$ are disregarded. Analogous expressions hold for $\frac{\partial^{2}}{\partial r_{2}^{2}}$ and $\frac{\partial^{2}}{\partial r_{3}^{2}}$ needed in the Laplacian occurring in (12) and (15). For 2-dimensional problems, the discretization error can be reduced to the order $\delta l^{8}$ if (16) is replaced by an expression involving four more neighbors [17]. The spa- 
tially discretized version of $W_{\mu}=0$ with $W_{\mu}$ given by (12) is

$\gamma_{1} \frac{\partial}{\partial t} n_{\mu}(\boldsymbol{i})-\mu_{0}^{-1} \chi_{\mathrm{a}} B_{\mu} B_{v} n_{v}(\boldsymbol{i})-K \delta l^{-2} D_{\mu}^{\mathrm{T}}(\boldsymbol{i})=0$,

where $D_{\mu}^{\mathrm{T}}$ is an abbreviation for

$$
\begin{aligned}
D_{\mu}^{\mathrm{T}}\left(i_{1}, i_{2}, i_{3}\right)=\{ & n_{v}\left(i_{1}+1, i_{2}, i_{3}\right) n_{\mu}\left(i_{1}+1, i_{2}, i_{3}\right) \\
& \left.+n_{v}\left(i_{1}-1, i_{2}, i_{3}\right) n_{\mu}\left(i_{1}-1, i_{2}, i_{3}\right)\right\} \\
& \cdot n_{v}\left(i_{1}, i_{2}, i_{3}\right)-2 n_{\mu}\left(i_{1}, i_{2}, i_{3}\right)+\ldots
\end{aligned}
$$

The ellipses in (18) stand for the analogous terms involving $i_{2} \pm 1$ and $i_{3} \pm 1$ corresponding to the spatial derivatives with respect to $r_{2}$ and $r_{3}$ needed for twoand three-dimensional problems, respectively.

The spatial discretization of $W_{\mu}^{\mathrm{V}}=0$, cf. (15), leads to an equation like (17) but with $D_{\mu}^{\mathrm{T}}$ replaced by

$$
\begin{aligned}
D_{\mu}^{\mathrm{V}}\left(i_{1}, i_{2}, i_{3}\right)= & n_{\mu}\left(i_{1}+1, i_{2}, i_{3}\right)+n_{\mu}\left(i_{1}-1, i_{2}, i_{3}\right) \\
& -2 n_{\mu}\left(i_{1}, i_{2}, i_{3}\right)+\ldots
\end{aligned}
$$

Again, the ellipses stand for the analogous terms involving $i_{2} \pm 1$ and $i_{3} \pm 1$. The superscripts " $T$ " and "V" in (18) and (19) refer to "tensorial" and "vectorial" since (18) preserves the tensorial symmetry. If the variation from one grid point to its neighbors is small, one has $n_{v}\left(i_{1}, i_{2}, i_{3}\right) n_{v}\left(i_{1} \pm 1, i_{2}, i_{3}\right) \approx 1$, and $D_{\mu}^{\mathrm{T}}$ reduces to $D_{\mu}^{\mathrm{V}}$. On the other hand, for directors which enclose angles $>90^{\circ}$ at adjacent grid points, the difference between (18) and (19) is really crucial. In particular, antiparallel neighbors are treated like parallel ones according to (18), but not to (19).

For a given initial condition, the first order differential equation (17) can be integrated by standard techniques. The simplest approximation is the replacement

$$
\frac{\partial}{\partial t} \rightarrow \delta t^{-1}\left(n_{\mu}^{\mathrm{ncw}}-n_{\mu}\right),
$$

where $n_{\mu}^{\text {ncw }}$ is the value of $n_{\mu}$ at the next time step $t+\delta t$. This leads to the iterative algorithm

$n_{\mu}^{\text {new }}=i\left\{n_{\mu}+\delta t \gamma_{1}^{-1}\left[K \delta l^{-2} D_{\mu}^{\mathrm{T}}+\mu_{0}^{-1} \chi_{\mathrm{a}} n_{v} B_{v} B_{\mu}\right]\right\}$,

where it is understood that $n_{\mu}$ and $D_{\mu}^{\mathrm{T}}$ are to be taken at the lattice points $i_{1}, i_{2}, i_{3}$, and $\lambda$ is determined by the constraint $n_{\mu} n_{\mu}=1$.

With the abbreviations

$$
\begin{aligned}
& \alpha=\delta t \gamma_{1}^{-1} K \delta l^{-2}=\frac{\delta t}{\tau_{\alpha}}\left(\frac{\xi_{0}}{\delta l}\right)^{2}, \\
& \alpha \beta=\delta t \gamma_{1}^{-1} \mu_{0}^{-1} \gamma_{\mathrm{a}} B^{2}
\end{aligned}
$$

(20) can be rewritten as

$$
n_{\mu}^{\text {new }}=\lambda\left\{(1-2 d \alpha) n_{\mu}+\alpha\left[2 d\left\langle n_{\mu} n_{v}\right\rangle+\beta \hat{B}_{\mu} \hat{B}_{v}\right] n_{v}\right\},
$$

where $\left\langle n_{\mu} n_{v}\right\rangle$ is the average over all $2 \mathrm{~d}$ nearest neighbors on a $d$-dimensional rectangular lattice and $\hat{\mathbf{B}}$ is a unit vector parallel to the magnetic field $\mathbf{B}$.

This equation shows clearly that the action of the nematic environment $\left\langle n_{\mu} n_{v}\right\rangle$, considered as a mean field, on a "computational molecule" is of the same (tensorial) types as the action of an external field $\mathbf{B}$.

Fastest convergence is achieved by the choice of $2 d \alpha=1$. This implies a time scaling of

$$
\delta t=\frac{\gamma_{1} \delta l^{2}}{2 d K}=\frac{1}{2 d} \tau_{\mathrm{a}}\left(\frac{\delta l}{\xi_{0}}\right)^{2},
$$

and (22) reduces to

$$
n_{\mu}^{\text {new }}=i\left\{\left\langle n_{\mu} n_{v}\right\rangle+\frac{\beta}{2 d} \hat{B}_{\mu} \hat{B}_{v}\right\} n_{v} ;
$$

$\beta$ depends on the material parameters and the length scale like

$$
\beta=\frac{\chi_{\mathrm{a}} \delta l^{2}}{\mu_{0} K} \cdot B^{2}=\operatorname{sign}\left(\chi_{\mathrm{a}}\right) \frac{\delta l^{2}}{\xi_{B}^{2}},
$$

where the magnetic coherence length $\xi_{B}$ is defined by $[1,2]$

$$
\xi_{B}^{-2}=\mu_{0}^{-1}\left|\chi_{\mathrm{a}}\right| B^{2} K^{-1} .
$$

For nematics with $\beta<0$, i.e. with a negative anisotropy of the magnetic susceptibility, $\chi_{\mathrm{a}}<0$, however, a modification is needed for reasons of numerical stability. More specifically, (22) is rewritten as

$$
\begin{aligned}
n_{\mu}^{\text {new }}= & \lambda\left\{(1-2 d \alpha+\alpha \beta) n_{\mu}+\alpha 2 d\left\langle n_{\mu} n_{v}\right\rangle\right. \\
& \left.-\alpha \beta\left(n_{\mu}-\hat{B}_{\mu} \hat{B}_{v} n_{v}\right)\right\} .
\end{aligned}
$$

Here, the negative action of the magnetic susceptibility on the vector $\hat{B}_{\mu} \hat{B}_{v} n_{v}$ is substituted by a positive action on the complementary vector $\left(\chi_{\mathrm{a}}<0\right.$ leads to $-\alpha \beta>0)$. The choice of $(1-2 d \alpha+\alpha \beta)=0$ yields fastest convergence; but now, the implied time scaling depends on the strength of the external field like

$$
\delta t=\frac{\gamma_{1} \delta l^{2}}{2 d K-\gamma_{\mathrm{a}} B^{2} \delta l^{2}},
$$

which means that strong fields cause an effective slowing down of the iteration.

For an electric field $\mathbf{E}$ instead of the magnetic field B, $\mu_{0}^{-1}$ and $\chi_{a}$ have to be replaced by the dielectric 
permeability coefficient $\varepsilon_{0}$ of the vacuum and by $\varepsilon_{\mathrm{a}}$, the anisotropy of the relative dielectric coefficient, respectively. In the absence of an external field one has $\beta=0$.

\subsection{Free Energy}

The stationary solution of (5) corresponds to a (local) minimum of the free energy $F=\int f_{\mathrm{a}} \mathrm{d} r^{3}$, with the free energy density

$f_{\mathrm{a}}=\frac{\varrho}{m} k_{\mathrm{B}} T\left[\phi-F_{\mu \nu} a_{\mu \nu}+\frac{1}{2} \xi_{0}^{2}\left(\nabla_{\lambda} a_{\mu \nu}\right)\left(\nabla_{\lambda} a_{\mu \nu}\right)\right]$.

As indicated in (6), the quantity $\Phi_{\mu \nu}$ occurring in (5) is the derivative of $\phi$ with respect to the alignment tensor $a_{\mu \nu}$. For a uniaxial alignment with constant order parameter $a_{\mathrm{eq}},(29)$ reduces to

$$
f_{\mathrm{a}}=\frac{\varrho}{m} k_{\mathrm{B}} T \phi+f_{\mathrm{a}}^{\text {or }},
$$

where the orientational part of the free energy density is

$$
\begin{aligned}
& f_{\mathrm{a}}^{\text {or }}=-\frac{1}{2} \mu_{0}^{-1} \chi_{\mathrm{a}} \overleftrightarrow{B_{\mu}} \vec{B}_{v} \overleftrightarrow{n_{\mu}} n_{v} \\
& +\frac{1}{4} K \nabla_{\lambda}\left(n_{\mu} n_{v}\right) \nabla_{\lambda}\left(n_{\mu} n_{v}\right) \text {. }
\end{aligned}
$$

Due to $\nabla_{\lambda}\left(n_{\mu} n_{v}\right) \nabla_{\lambda}\left(n_{\mu} n_{v}\right)=2 \nabla_{\lambda} n_{\mu} \nabla_{\lambda} n_{\mu}$, (31) is equivalent to the standard expression for $f_{\mathrm{a}}^{\text {or }}$ in the one-coefficient approximation [1-3]. The factors occurring in (8) and (13) have been chosen such that (31) follows from (29).

If the first spatial derivative $\frac{\partial}{\partial r_{1}}$ of a function $G$ is
scretized according to

$$
\frac{\partial}{\partial r_{1}} \mathrm{G} \approx \delta l^{-1}\left[G\left(i_{1}+1, i_{2}, i_{3}\right)-G\left(i_{1}, i_{2}, i_{3}\right)\right],
$$

the discretized version of the integral over (31) is

$$
\int f_{\mathrm{a}}^{\text {or }} \mathrm{d} r^{3}=-\frac{K \delta l}{2} \sum_{i_{1}} \sum_{i_{2}} \sum_{i_{3}}\left[Y^{\mathrm{T}}-\beta\left(\hat{B}_{\mu} n_{v}\right)^{2}\right]
$$

with

$$
\begin{aligned}
Y^{\mathrm{T}}\left(i_{1}, i_{2}, i_{3}\right)=d & -\left[n_{\mu}\left(i_{1}+1, i_{2}, i_{3}\right) n_{\mu}\left(i_{1}, i_{2}, i_{3}\right)\right]^{2} \\
& -\ldots,
\end{aligned}
$$

where $d$ is the dimension and the ellipses stand for analogous terms involving $i_{2}+1$ and $i_{3}+1$ needed for two- and three-dimensional problems. The energy equation (33) refers to a liquid crystal with the size $\delta l$ in each omitted dimension and has therefore to be multiplied by a suitable factor to match the desired

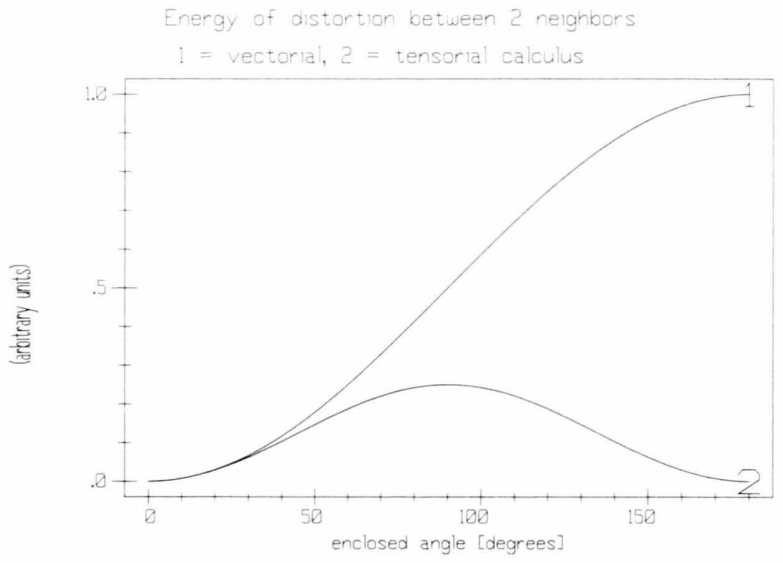

Fig. 1. Tensorial and vectorial form of the anisotropic energy between a pair of computational molecules. Only for small angles, the difference is negligible.

size. Discretization of the vectorial expression $\nabla_{\lambda} n_{\mu} \nabla_{\lambda} n_{\mu}$ leads to (32) with $Y^{\mathrm{T}}$ replaced by

$Y^{\mathrm{V}}\left(i_{1}, i_{2}, i_{3}\right)=d-\left(n_{\mu}\left(i_{1}+1, i_{2}, i_{3}\right) n_{\mu}\left(i_{1}, i_{2}, i_{3}\right)\right)-\ldots$,

with the ellipses meaning the same as in (33). Again, for $n_{\mu}\left(i_{1}+1, i_{2}, i_{3}\right) \approx n_{\mu}\left(i_{1}, i_{2}, i_{3}\right)$, (33) reduces to (34). However, for neighbors which enclose angles larger than about $90^{\circ}$ the difference between (33) and (34) is significant. This can also be inferred from Fig. 1 where $Y^{\mathrm{T}}$ and $Y^{\mathrm{V}}$ are displayed (for a one-dimensional spatial dependence) as functions of the angle $\psi$ between directors at neighboring sites.

\section{Applications}

\subsection{Relaxation after a Temperature Quench}

The first application of the algorithm is the simulation of the relaxation process of a nematic liquid crystal beginning with an isotropic director configuration. This describes the alignment after a fast cooling down from the isotropic state.

The iteration was performed on a two-dimensional lattice with $21 \times 21$ grid points and homeotropic boundary conditions. The interior vectors $\mathbf{n}(\boldsymbol{i})$ were produced with the aid of a random number generator and a suitable algorithm that provides an isotropic distribution.

Figure 2 shows the director configuration obtained with the usual vectorial algorithm, cf. (17) and (19). It leads, like the corresponding differential equations 


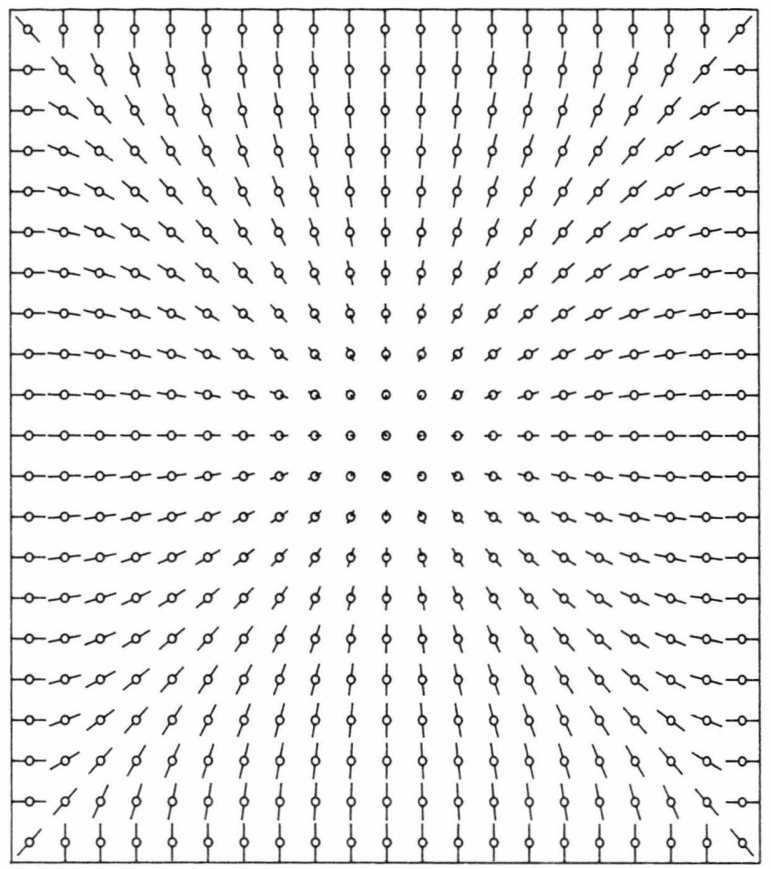

Fig. 2. Equilibrium director configuration for a square with homeotropic boundary conditions, vectorial calculus. The director field escapes into the third dimension.
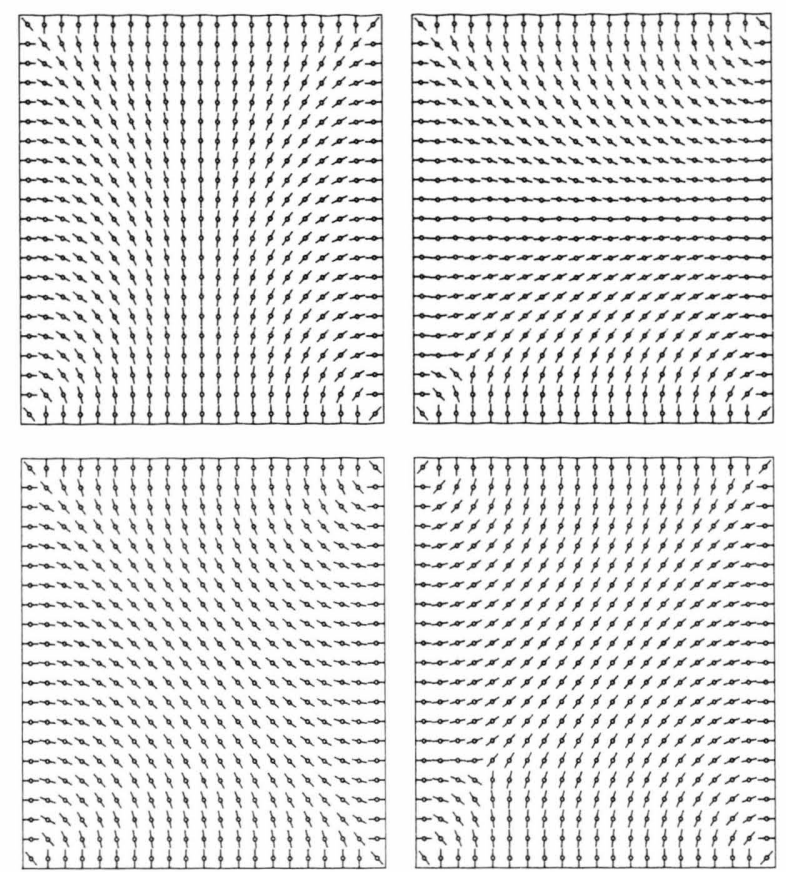

Fig. 3. Equilibrium director configurations obtained after 1000 iterations with the tensorial algorithm. In Figs. $3-5$ the iterations started with different random isotropic initial configurations. Preferential orientations and the number of defects (with half integer winding numbers) depend sensitively on the initial conditions.

$(3,7,6,8),(16,17,9,20)$ and $(14,19,11,1)$ they have low, medium, and high anisotropic energies, respectively, cf. Fig. 6. Similar to real nematics, the director field is not steady, but disclinations occur. It should be mentioned that the configuration from Fig. 2 is also a stable solution of the tensorial algorithm but in connection with the applied initial conditions a rather improbable one. The states shown in Figs. 2-5 were reached after 1000 iteration steps. The directors in the corners of the squares were not used in the calculation but added in the figures for aesthetic reasons.

Figure 6 exhibits the effect of the disclinations on the anisotropic energy, cf. (33). Most of the director configurations with disclinations have energies lower than the smooth one shown in Figure 2.

However, the energy of real disclinations cannot be deduced in this way because the size of the disclinations occurring in the computer-simulation is comparable to the lattice-spacing $\delta l$, whereas the real disclination core is rather within the range of molecular dimensions, which causes quite different energies. 

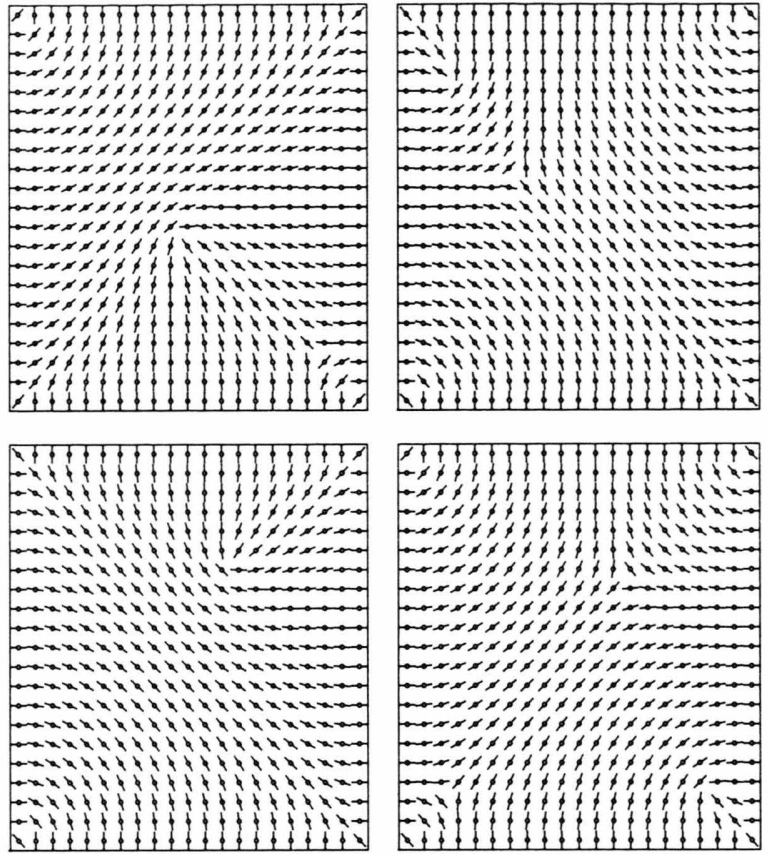

Fig. 4. Same as Fig. 3 but with different random initial configurations.
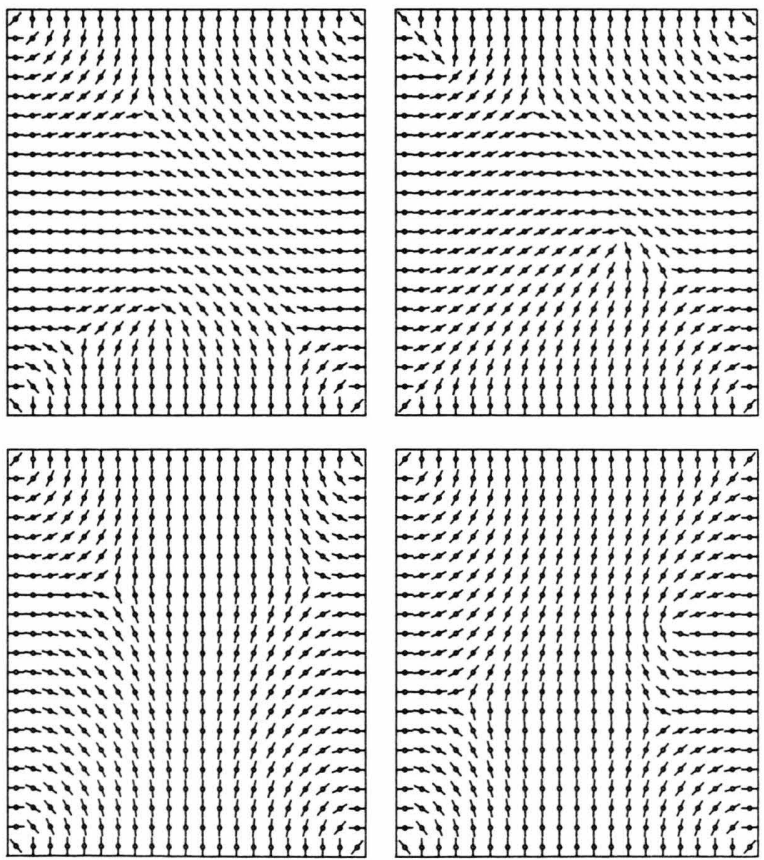

Fig. 5. Same as Figs. 3 and 4 with different random initial configurations.

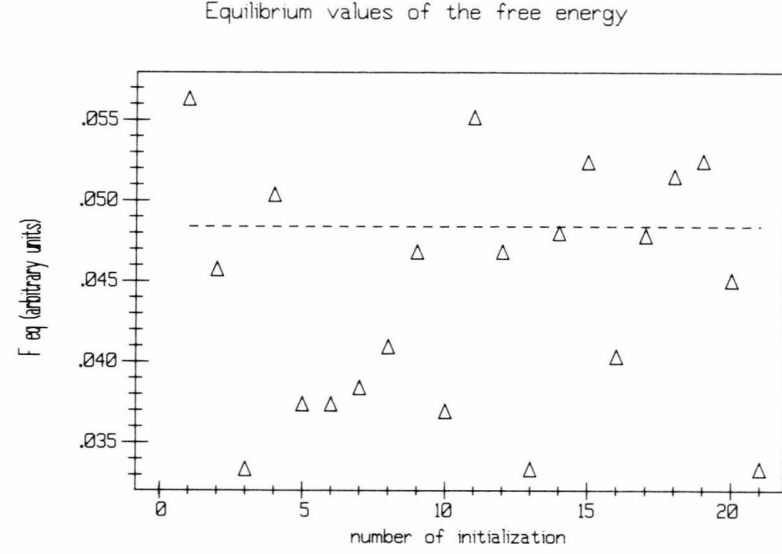

Fig. 6. The equilibrium anisotropic energies of different director configurations found after 1000 iterations starting from different random initial configurations labeled by the numbers 1 to 21. The final configurations shown in Fig. 3 correspond to the numbers 6, 8, 3, 7; those of Fig. 4 to 9, 20, 16, 17, and those of Fig. 5 to the initialization numbers 11, 1 , 14, 19. Dashed, the energy of Fig. 2 (vectorial calculus).

Furthermore, a possible biaxiality and a spatial variation of the order parameter is not taken into consideration here [18].

\subsection{Simulation of a Frederiks Cell}

This example is meant to check the reliability of the program with respect to the Frederiks-threshold, to demonstrate the dynamical behavior of the relaxation process, and to examine the effect of weak anchoring on the response function to an external field

The calculations were done in one dimension, which is well applicable to usual Frederiks cells, where the thickness of the nematic layer is small compared to the cell dimensions, and where the applied field prevents disclinations, such that the director field may be considered homogeneous in the planes parallel to the anchoring surfaces of the cell.

\subsubsection{Director Profile}

The Frederiks cell is characterized by parallel boundary conditions and an external field perpendicular to the boundaries. The coordinates need not to be specified here because the three Franck elastic constants are assumed equal. The case $\beta>0\left(\chi_{\mathrm{a}}>0\right)$ was considered, where the director wants to be parallel to the applied field. Some technical details on the calculation of the equilibrium profile are in order. First, the threshold field was determined by a routine which 


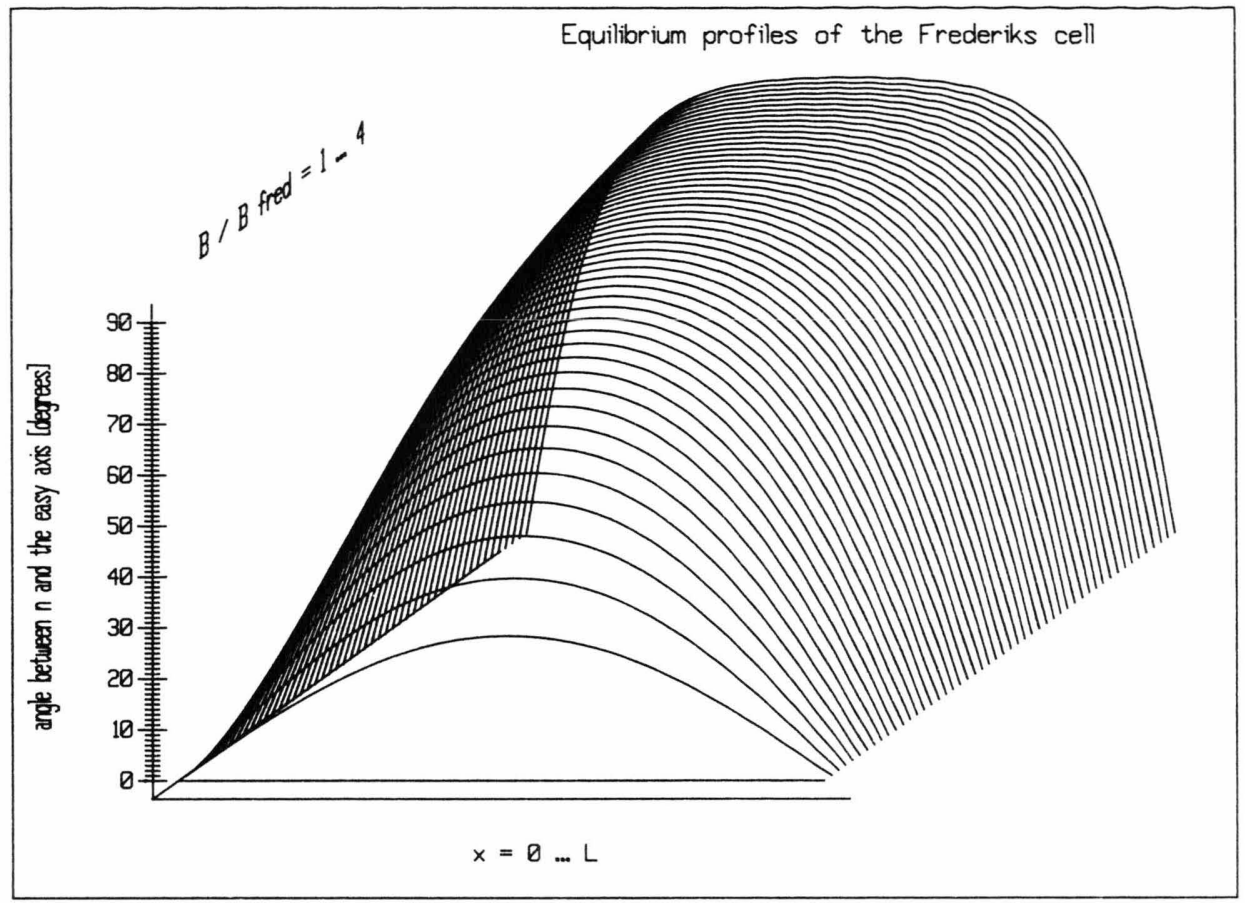

Fig. 7. Equilibrium director profiles of a (one-dimensional) Frederiks cell for various field strengths $B>B_{\text {fred }}$ as a 3 D-Plot. Even for angles up to $70^{\circ}$, the profile is sine-shaped.

searches for the field where the maximum value of the component of the director parallel to the applied field has a prescribed small but nonzero equilibrium value (e.g. $\left.10^{-3}\right)$ with a prescribed variance $\left( \pm 10^{-5}\right)$. Then the field strength was increased in small steps and the equilibrium profiles were calculated for the various values of the field. Every $100^{\text {th }}$ time step, the director field was compared with the previously stored configuration; if the change of any component at any site was smaller or equal a prescribed variance (typically $10^{-5}$ ), the iteration was terminated.

Analytically, the behavior of the Frederiks cell slight above the threshold field, $B_{\text {fred }}$, can be found by the use of the "small angle approximation", which yields, in the limit of small distortions, a sine-shaped spatial dependence of the angle $\phi$ between the director $\mathbf{n}$ and the easy direction.

Figure 7 shows the director profile of a Frederiks cell for an external field strength $B=1 \ldots 4 B_{\text {fred }}$. It is to be seen that the small angle approximation even holds for distortion angles $\approx 70^{\circ}$, corresponding to a range of the applied field up to twice the Frederiks threshold.
3.2.2 Dynamics of the Relaxation Process at Different Field Strengths

It is well known that the director profile of a Frederiks cell as a function of the external field describes a phase transition of the first kind [1,2], with a critical value $B_{\text {fred }}$ below which the applied field does not affect the nematic layer: $B_{\mathrm{fred}}$ depends on the material properties like

$$
B_{\text {fred }}=\mu_{0} \cdot \frac{\pi}{L} \cdot\left(\frac{K}{\chi_{\mathrm{a}}}\right)^{1 / 2},
$$

$\chi_{\mathrm{a}}>0$ and strong anchoring provided. With it, (25) can be generalized to

$$
\beta=\left(\frac{\pi \delta l}{L}\right)^{2} \cdot\left(\frac{X}{X_{\mathrm{fred}}}\right)^{2},
$$

where the generalized force $X$ is either an electric or a magnetic field strength.

Figure 8 shows the number of time steps needed for the relaxation process as a function of the field strength. As an example, inserting the material parameters of a typical room temperature nematic 
Number of time steps needed for the relaxation

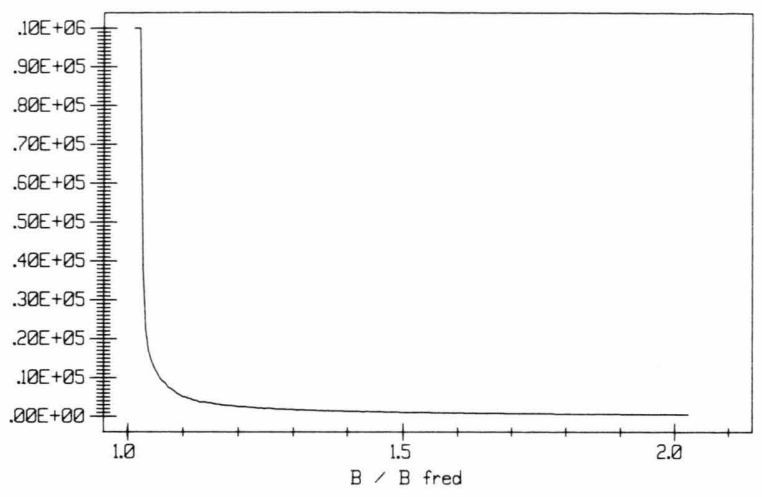

Fig. 8. Number of iteration time steps needed to reach the equilibrium state as a function of the applied field strength. The relaxation time diverges for $B \rightarrow B_{\text {fred }}$ (critical slowing down).

such as MBBA or $5 \mathrm{CP}, \quad \gamma_{1} \approx 0.1$ Pas and $K \approx 10 \cdot 10^{-12} N$ into (23), assuming a cell thickness of $L=20 \mu \mathrm{m}$ and a lattice spacing of $\delta l=250 \mathrm{~nm}$ one obtains a time step of $\delta t \approx 0.3 \mathrm{~ms}$.

The divergence of the relaxation time at the critical field is well known as "critical slowing down". Generally, the relaxation time depends on the relative difference from the critical point $X_{\text {crit }}$ like [17]

$$
\tau=C \cdot\left(\frac{X}{X_{\text {crit }}}-1\right)^{\gamma}
$$

with $C$ a constant depending on the units and $X$ a generalized force; $\gamma$ depends on the special type of the phase transition. Here, it was found that (36) applies with $\gamma=-1$. This is evident from Fig. 9 where the inverse number of the iterations needed at different field strength $B>B_{\text {fred }}$ is displayed as a function of $B / B_{\text {fred }}-1$. The steps in the curve at high field strengths are due to the fact that the equilibrium condition in the computer program was checked every $100^{\text {th }}$ iteration.

Referring to the speed of the relaxation process, it is not surprising that the iteration exhibits an exponential slowing down, at least near the equilibrium. Figure 10 shows the speed of the iteration (defined as the maximum of the change of any component of the director at any site) for a two-dimensional liquid crystal as e.g. in Figs. 2-5 in absence of external fields as a function of the iteration number, i.e. its time dependence. The irregularities are due to the random "isotropic" starting condition.

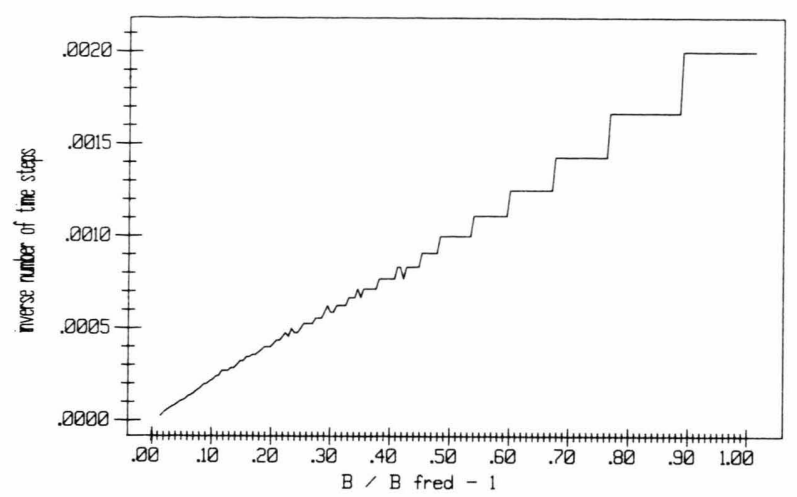

Fig. 9. Same information as in Fig. 8, but now the inverse number of time steps is plotted versus $B / B_{\mathrm{fred}}-1$.

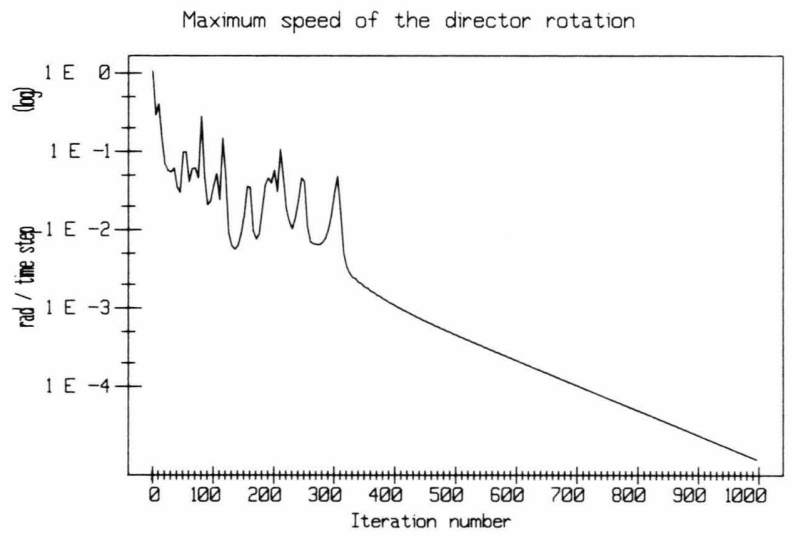

Fig. 10. The speed of the iteration for the relaxation of a two-dimensional liquid crystal which leads to a state as shown in Figs. 3-5 is an exponential function (log. scale) of the number of iterations performed after the initialization.

\subsubsection{Frederiks-Threshold of a Cell with Weak Anchoring}

The physics of the anchoring process, i.e. the interaction between the walls and the nematic fluid, is not yet well understood. Different forms of boundary conditions have been proposed [19].

Here, the weak anchoring was realized by altering the length of the boundary vectors, $\left|\mathbf{n}^{\mathrm{w}}\right|<1$. This could either be understood as the influence of an intermediate layer near the wall with an elastic constant $K^{\mathrm{W}}=\left|\mathbf{n}^{\mathrm{W}}\right| \cdot K$, where $K$ is the Frank elastic constant in the bulk. Alternatively, irregularities of the an- 


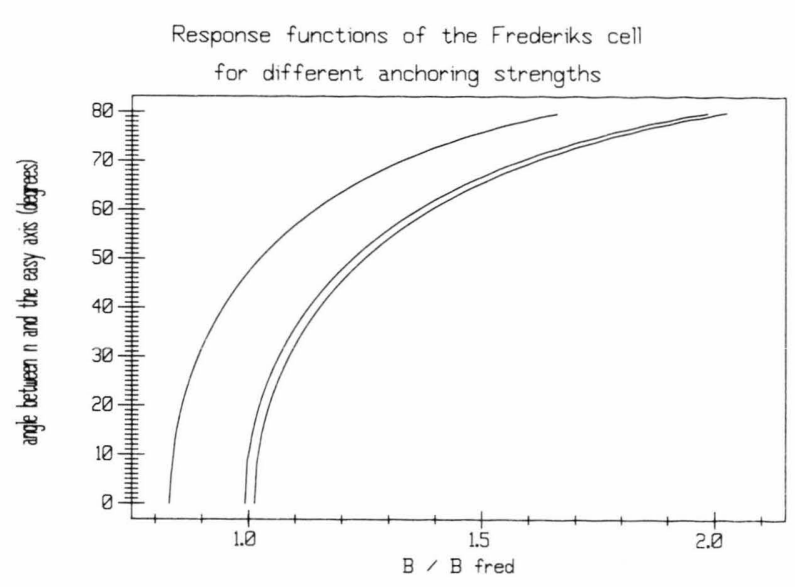

Fig. 11. Response functions for weak, medium, and strong anchoring $\left.K_{\mathrm{w}} / K \equiv\left|\mathbf{n}^{\mathrm{w}}\right|=0.1 / 0.55 / 1.0\right)$. Diminishing the anchoring strength causes a shift of the response function without altering its shape.

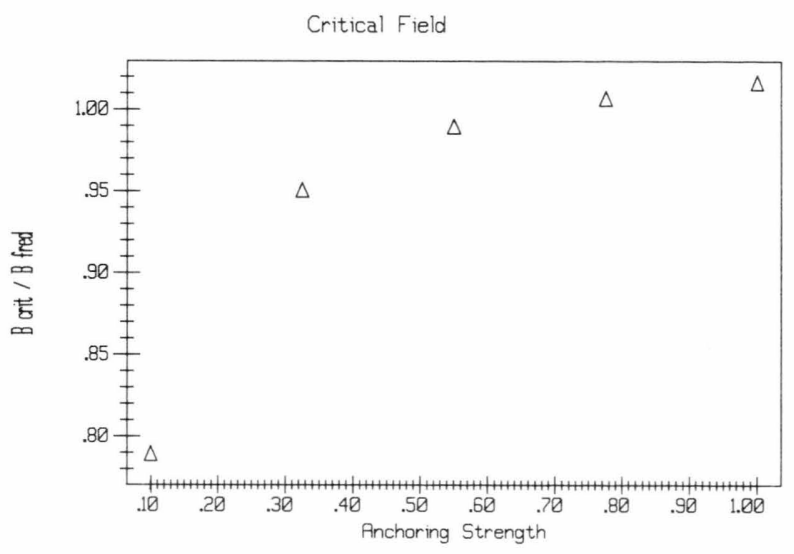

Fig. 12. Influence of the coupling constant between the bulk and the boundary on the threshold field, for a thickness of the wall layer $R=1 / 80$ of the cell thickness.

choring surface may diminish the order parameter $a$ at the boundary and therefore the length of $\mathbf{n}^{\mathrm{w}}$, cf. (3), (4).

To examine the effect of weak anchoring on the director profile it is sufficient to focus on the center of the nematic layer. The angle between the director field in the middle of the cell and the easy direction will be referred to as the response function. Figure 11 shows the response functions for different degrees of anchoring, $\left|\mathbf{n}^{\mathrm{w}}\right|=0.1 \ldots 1$. The applied field strength is measured in units of the theoretical value of the threshold field, (35). The response function for $\left|\mathbf{n}^{\mathrm{w}}\right|=1$ (strong anchoring) should meet the $x$-axis at $B$
$B_{\mathrm{fred}}=1$. Slight differences are due to the discretization error (a field length of 80 was used) and to the finite computing time.

The first result is, that weak anchoring causes a shift of the response function but practically does not alter it's shape. Second, the coupling constant between the bulk and the boundary has not much influence on the threshold field, cf. Fig. 12, at least for the ratio $R / L=1 / 80$ used here. In experiments, the thickness $R$ of the wall layer was found to be in the range of $0.3 \mu \mathrm{m}$ [20], so that the data obtained here are appropriate for cells with $L \approx 20 \mu \mathrm{m}$.

\section{Concluding Remarks}

Starting from a relaxation equation for the alignment tensor, an algorithm has been derived which allows the numerical calculation of the dynamic and static behavior of the director field $\mathbf{n}$ with the correct nematic symmetry property where $\mathbf{n}$ and $-\mathbf{n}$ are equivalent. So far, the special case of the one-coefficient approximation for the Frank elastic energy has been treated. The generalization to two different coefficients, in particular the case where one has $K_{1}=K_{3} \neq K_{2}$ is straight forward. For the general case of three different elasticity coefficients $K_{1}, K_{2}$, $K_{3}$ one has to start from equations which also involve the $4^{\text {th }}$ rank alignment tensor [13].

The extension to cholesterics is desirable and seems to be feasible.

The applications considered were meant to show the difference between the conventional vectorial algorithm and the new tensorial one which is essential for the occurrence of the defects typical for nematics, as displayed in Figs. 3-5, and, secondly, the applicability of the method to a quantitative analysis of the static and dynamic response of the local orientation subjected to an external field. So far, a Frederiks transition was studied. In view of the interest for display applications, the study of the $\pi$-cell and analogous devices [21] as well as of supertwisted cells is desirable. Furthermore, the director field under the influence of a strong laser field as observed in nonlinear optical experiments [22] can be treated. For the direct comparison with the experiments, also the optical properties have to be calculated from the director field. A simple modification of the boundary layer was considered here. The method also allows a more systematic analysis of the effect of modified boundary conditions, 
(e. g. caused by scratches, imperfections, and modulations of the anchoring of the molecules of a surface) on the behavior of a nematic cell.

\section{Acknowledgements}

Financial support by the Deutsche Forschungsgemeinschaft via the Sonderforschungsbereich "Anisotrope Fluide" (SFB 335) is gratefully acknowl-

[1] P. G. de Gennes, The Physics of Liquid Crystals, Clarendon Press, Oxford 1974; - M. J. Stephen and J. P. Straley, Rev. Mod. Phys. 46, 617 (1974).

[2] H. Kelker and R. Hatz, Handbook of Liquid Crystals, Verlag Chemie, Weinheim 1980.

[3] G. Vertogen and W. H. de Jeu, Thermotropic Liquid Crystals, Fundamentals, Springer, Berlin 1988.

[4] G. Baur, The Physics and Chemistry of Liquid Crystal Devices, ed. G. Sprokel, p. 61, Plenum Press, New York 1980; Mol. Cryst. Liq. Cryst. 63, 45 (1981); - D. W. Berremann, Phil. Trans. Roy. Soc. London A. 309, 203 (1983).

[5] H. Wöhler, G. Haas, M. Fritsch, and D. A. Mlyski, Proc. 17. Freiburger Arbeitstagung Flüssigkristalle 1987; I. Heynderickx and H. de Raedt, Phys. Rev. A 37, 1725 (1988).

[6] S. Y. Lin and M. Laskin, Relaxation Methods for Liquid Crystal Problems, IAM reprint series 332, Minneapolis, 1987.

[7] W. Zimmermann and L. Kramer, Phys. Rev. Lett. 56, 2655 (1986); - L. Kramer, E. Bodenschatz, W. Pesch, and W. Zimmermann, in: The Physics of Structure Formation, eds. W. Güttinger and G. Danglmayer, Springer, Berlin 1987.

[8] H. R. Trebin, Adv. Phys. 31, 195 (1982).

[9] S. Hess, Z. Naturforsch. 30a, 728; 1224 (1975); 31 a, 1507 (1976) edged. We thank Prof. Dr. W. Muschik, Dipl. Phys. R. Ellinghaus, Dipl. Phys. R. Macdonald, Dipl. Ing. G. Gleske (Technische Universität Berlin) and Dipl. Phys. G. Haas (Institut für theoretische Elektrotechnik Karlsruhe) for helpful discussions. Part of the numerical calculations was performed on the HewlettPackard 9000/840 of the Prozessrechner-VerbundZentrale of the Technische Universität Berlin. We thank Dr. K. Rebensburg and his staff for their advice.

[10] S. Hess and I. Pardowitz, Z. Naturforsch. 36a, 554 (1981).

[11] S. Hess, J. Non-Equilib. Thermodyn. 11, 176 (1986).

[12] S. Hess, Z. Naturforsch. 31 a, 1034; 1224 (1976).

[13] I. Pardowitz and S. Hess, J. Chem. Phys. 76, 1485 (1982).

[14] P. J. Wojtowicz and P. Sheng, Phys. Lett. 48 a, 235 (1974).

[15] W. Jäger and H. Kaul, J. reine u. angew. Math. 343, 146 (1983).

[16] S. Blenk and W. Muschik, Technische Universität Berlin, to be published (1989).

[17] A. I. van de Vooren and A. C. Vliegenthart, Report TW-42, Math. Institute, University of Grooningen, 1967.

[18] N. Schopohl and T. J. Sluckin, Phys. Rev. Lett. 59, 2582 (1987).

[19] G. Barbero and C. Oldano, Nuovo Cim. Serie 1, 6d, 479 (1985).

[20] H. Wöhler, G. Haas, M. Fritsch, and D. A. Mlyski, Proc. $12^{\text {th }}$ Liquid Crystal Conference, p. 401, Freiburg 1988.

[21] P. J. Bos and K. R. Koehler, Mol. Cryst. Liq. Cryst. 113, 329 (1984).

[22] H. J. Eichler and R. Macdonald, Proc. 18. Freiburger Arbeitstagung Flüssigkristalle 1989. 